\title{
Avaliação de parâmetros dosimétricos de uma fonte de Braquiterapia em regiões próximas à fonte
}

Evaluation of dosimetric parameters for brachytherapy source in regions close to the source

\author{
A. Quevedo ${ }^{1 *} ;$ L. F. Borges ${ }^{2} ;$ P. Nicolucci ${ }^{1}$ \\ ${ }^{1}$ Departamento de Física, Universidade de São Paulo - Faculdade de Filosofia, Ciências e Letras de Ribeirão Preto, \\ 14040-901, Ribeirão Preto-São Paulo, Brasil \\ ${ }^{2}$ Departamento de Radioterapia, Hospital das Clinicas da Faculdade de Medicina de Ribeirão Preto - Universidade de \\ São Paulo, 14040-901, Ribeirão Preto-São Paulo, Brasil \\ *anaquevedo@usp.br \\ (Recebido em 27 de fevereiro de 2018; aceito em 30 de abril de 2018)
}

\begin{abstract}
Neste trabalho foram determinadas as funções dosimétricas de uma fonte clínica de braquiterapia de alta taxa de dose de Irídio-192 em distâncias próximas à fonte. O fator geométrico foi determinado analiticamente, enquanto que a função de dose radial e a função de anisotropia foram determinadas para distâncias de até $5 \mathrm{~cm}$ do centro da fonte utilizando simulação Monte Carlo com o pacote PENLEOPE. Uma fonte de ${ }^{192} \mathrm{Ir}$ de alta taxa de dose (Varian, modelo GammaMed Plus) foi modelada e posicionada no centro de um objeto simulador cúbico de $30 \mathrm{~cm}$ de lado e preenchido com água. Foi realizada a paralelização do pacote PENELOPE para a realização das simulações, utilizando 8 processadores, onde o número de partículas primárias em cada processador permaneceu constante em $10^{9}$, a energia de corte para fótons e elétrons foi de $100 \mathrm{keV}$ e os parâmetros de condensação de histórias ( $\mathrm{C} 1$ e C2) foram de 0,3 , otimizando o tempo de simulação e não adicionando incertezas sistemáticas aos resultados. Perfis de dose relativa obtidos utilizando simulação Monte Carlo foram comparados com o sistema de planejamento BrachyVision em três diferentes planos axiais em relação à fonte: plano central; $0,4 \mathrm{~cm}$ acima da fonte, na direção do topo do encapsulamento; e $0,4 \mathrm{~cm}$ abaixo da fonte, na direção do cabo. Quando comparados entre si, ambos os conjuntos de dados possuem o mesmo comportamento, com diferenças máximas de $2 \%$, indicando que o pacote PENELOPE apresenta-se como ferramenta promissora em cálculos dosimétricos de fontes de braquiterapia de alta taxa de dose.
\end{abstract}

Palavras-chave: Braquiterapia, Simulação Monte Carlo, pacote PENELOPE

In this work were determined the dosimetric parameters for ${ }^{192}$ Ir HDR brachytherapy source in regions close to the source. The geometry function was determined analytically, while the radial dose function and anisotropy function were determined in distances up to $5 \mathrm{~cm}$ from the center of the source using Monte Carlo simulation with PENELOPE package. One source of 192Ir with HDR (Varian, model GammaMed Plus) was modeled and placed in the center of the cubic phantom with $30 \mathrm{~cm}$ of side and filled with water. Were realized the parallelization of PENELOPE package to perform the simulation, where the number of primary particles in each processor remained constant in $10^{9}$, the cutoff energy of photons and electrons was $100 \mathrm{keV}$, and the condensed stories parameters $(\mathrm{C} 1$ and $\mathrm{C} 2)$ were 0.3 , optimizing the simulation time and do not adding systematic uncertainties to the results. Dose relative profiles obtained using Monte Carlo simulation were compared with BrachyVision treatment planning system in three different axial planes relative to the source: central plane; $0.4 \mathrm{~cm}$ above the source in the direction of encapsulation top, and $0.4 \mathrm{~cm}$ below the source, in the direction of the source cable. These results, when compared to each other, showed the same behavior, with maximum differences of $2 \%$, indicating that PENELOPE package presents as a promising tool in dosimetric calculations of high dose rate brachytherapy sources.

Keywords: Brachytherapy, Monte Carlo Simulation, PENELOPE package

\section{INTRODUÇÃO}

Sistemas de Planejamento tipicamente utilizados em Braquiterapia incorporam informações com relação a geometria da fonte radioativa e atenuação da radiação. As funções dosimétricas que compõem um sistema de planejamento de Braquiterapia são: Fator Geométrico, Função de Dose Radial e Função de Anisotropia. As funções foram descritas em um formalismo proposto pela Associação Americana de Físicos em Medicina em 1995, intitulado por TG-43 e revisado em 2004 
e chamado de TG-43U1 [1-2]. Os valores do fator geométrico são determinados analiticamente, enquanto que a função de dose radial e a função de anisotropia podem ser determinadas experimentalmente. No entanto, devido ao alto gradiente de dose em regiões próximas à fonte de radiação, devido à sua alta taxa de dose e produção de fótons de baixas energias, é recomendado o uso de simulação Monte Carlo para o cálculo desses parâmetros [3-11].

Atualmente, diversos pacotes de simulação são utilizados em aplicações em radioterapia, tais como GEANT [12], MCNP [13], PENELOPE [14], GATE [15]. Tais aplicações podem estar relacionadas à determinação de parâmetros dosimétricos, análise de espectros de fontes radioativas, cálculos de dose absorvida em determinados órgãos ou outros tipos de aplicações em radioterapia [16-20].

O pacote de simulação Monte Carlo PENELOPE simula o transporte de elétrons, pósitrons e fótons em materiais e geometrias arbitrárias, possuindo um arquivo de dados de seções de choque para os elementos da tabela periódica e outros 180 compostos e misturas de interesse em Física Radiológica [14]. O pacote PENELOPE implementa um algoritmo chamado classe II ou algoritmo misto, que realiza simulações detalhadas de fótons, calculando o ângulo de espalhamento e perda de energia em cada passo individual da partícula. Para simulações utilizando fótons, a simulação poderá ser realizada de forma detalhada, enquanto que para simulações onde forem utilizados elétrons ou pósitrons, a simulação é realizada através do processo misto, sendo detalhada para o caso de eventos onde ocorram interações fortes ou para espalhamentos fracos ela será realizada de forma condensada.

Nesse trabalho, foi utilizado o pacote PENELOPE para a determinação da Função de Dose Radial e Função de Anisotropia, enquanto o Fator Geométrico foi determinado analiticamente, para uma fonte de Irídio-192 utilizada em braquiterapia de alta taxa de dose. Foram determinados os parâmetros dosimétricos em regiões próximas à fonte de braquiterapia, até $5 \mathrm{~cm}$ do centro da mesma. Adicionalmente, os perfis de dose relativa obtidos utilizando simulação Monte Carlo com o pacote PENELOPE, foram comparados ao sistema de planejamento BrachyVision, a fim de se verificar a exatidão dos dados obtidos nesse trabalho.

\section{MATERIAL E MÉTODOS}

Nesse trabalho foram determinados os parâmetros dosimétricos de uma fonte de ${ }^{192} \mathrm{Ir}$ de Braquiterapia, Varian modelo GammaMed Plus, comumente usada em tratamentos ginecológicos. Essa fonte foi modelada utilizando o pacote PENELOPE para obtenção de parâmetros dosimétricos utilizados em planejamento de tratamentos.

$\mathrm{O}$ fator geométrico foi determinado analiticamente, enquanto a função de anisotropia e a função de dose radial foram determinadas usando simulação Monte Carlo com o pacote PENELOPE. Todos os parâmetros dosimétricos foram calculados para distâncias de $1,0 \mathrm{~cm}$ à $5 \mathrm{~cm}$ do centro da fonte. Adicionalmente, os valores de dose relativa na direção radial obtidos utilizando simulação Monte Carlo foram comparados com um sistema de planejamento de Braquiterapia em três diferentes planos axiais da fonte: plano central; a $0,4 \mathrm{~cm}$ acima da fonte, na direção do encapsulamento; e a $0,4 \mathrm{~cm}$ abaixo da fonte, na direção do cabo da mesma.

\section{Parâmetros dosimétricos em Braquiterapia}

De acordo com o protocolo para dosimetria em braquiterapia (TG-43 e posteriormente revisado, TG-43U1), uma distribuição bidimensional de dose pode ser calculada de acordo com a distância ao ponto de interesse $(r)[1,21]$ em relação ao centro da fonte e o ângulo de interesse $(\theta)$ em relação ao eixo transversal da fonte. Na figura 1 é apresentado um sistema de coordenadas tipicamente utilizado em dosimetria em braquiterapia e apresentado por esse protocolo. 


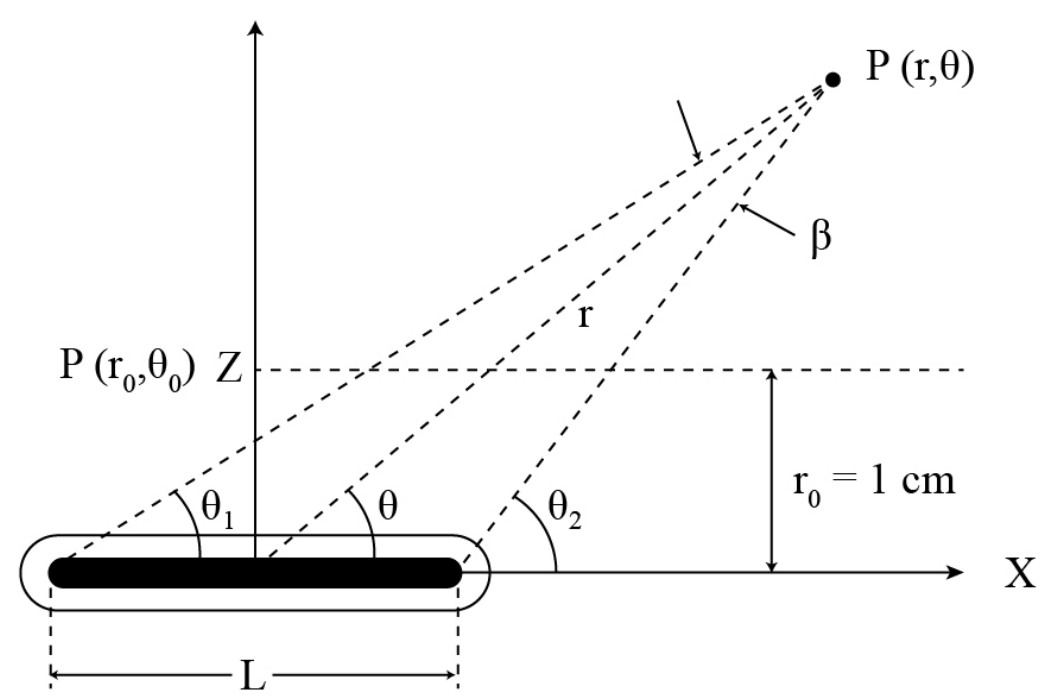

Figura 1: Sistema de coordenadas usado em cálculo de dosimetria em Braquiterapia [1].

De acordo com o formalismo para dosimetria em braquiterapia proposto pelo TG-43, a taxa de dose pode ser calculada a partir da equação (1):

$$
\dot{D}(r, \theta)=S_{K} \cdot \Lambda \cdot \frac{G_{L}(r, \theta)}{G_{L}\left(r_{0}, \theta_{0}\right)} \cdot g_{L}(r) \cdot F(r, \theta)
$$

onde $r_{0}$ é a distância de referência $(1 \mathrm{~cm}) ; r$ é a distância do centro da fonte ao ponto de interesse $(\mathrm{em} \mathrm{cm}) ; \theta$ é o ângulo polar que especifica o ponto de interesse; $\theta_{0}$ é o ângulo de referência que define o plano transverso da fonte $\left(90^{\circ}\right) ; \Lambda$ é a constante de taxa de dose (na água); $\mathrm{S}_{\mathrm{K}}$ é a intensidade de kerma no ar; $G_{L}(r, \theta)$ é o fator geométrico; $g_{L}(r)$ é a função de dose radial; e $F(r, \theta)$ é a função de anisotropia.

No cálculo da taxa de dose, bem como nos cálculos dos parâmetros dosimétricos, todos os parâmetros denotados com o subíndice " 0 " remetem à condição de referência e parâmetros sem subíndice referem-se à condição de interesse.

\section{Fator Geométrico}

O fator geométrico $\left[G_{L}(r, \theta)\right]$ considera a influência que a distribuição e geometria do material da fonte radioativa possuem na distribuição de dose. No entanto, ele não leva em consideração a absorção da radiação e o espalhamento que ocorre no encapsulamento ou no objeto simulador [1]. O fator geométrico utilizando uma aproximação de fonte linear é calculado de acordo com a equação (2):

$$
G_{L}(r, \theta)=\frac{\beta}{L r \operatorname{sen} \theta}
$$

onde $\beta$ é a subtração dos ângulos $\theta_{2}$ e $\theta_{1}$.

Diversas equações podem ser usadas para determinar $\beta$, porém devido à combinação de fatores trigonométricos, algumas podem apresentar descontinuidade em determinados ângulos. Nesse trabalho, foram utilizadas as equações apresentadas por Rivard, que não contém descontinuidade em nenhuma distância e nenhum ângulo de interesse [22]. As equações utilizadas nesse estudo para o cálculo do fator geométrico são mostradas em (3), (4) e (5):

$$
G_{L}=\frac{1}{r^{2}-(L / 2)^{2}}, \text { se } \theta=0^{o}
$$




$$
\begin{gathered}
G_{L}=\frac{2 \operatorname{arctg}(L / 2 r)}{L r} \text {, se } \theta=90^{\circ} \\
G_{L}=\frac{\operatorname{arctg}\left[\frac{L}{2 r \operatorname{sen} \theta}+\operatorname{cotg} \theta\right]+\operatorname{arctg}\left[\frac{L}{2 r \operatorname{sen} \theta}-\operatorname{cotg} \theta\right]}{\operatorname{Lrsen} \theta} \text {, se } 0<\theta<90^{\circ}
\end{gathered}
$$

Para a fonte clínica de ${ }^{192}$ Ir utilizada nesse estudo, o comprimento efetivo da fonte é de $0,35 \mathrm{~cm}$. Foram determinados os valores do fator geométrico em distâncias de $0,3 \mathrm{~cm}$ à $5,0 \mathrm{~cm}$ do centro da fonte, para ângulos de $0^{\circ}$ à $90^{\circ}$. Os valores de $90^{\circ}$ à $180^{\circ}$ não serão apresentados devido à simetria da fonte.

\section{Função de Dose Radial}

A função de dose radial $\left[g_{X}(r)\right]$ fornece informações sobre a dose no plano transverso da fonte. Matematicamente, essa função pode ser calculada através da equação (6):

$$
g_{X}(r)=\frac{\dot{D}\left(r, \theta_{0}\right)}{\dot{D}\left(r_{0}, \theta_{0}\right)} \frac{G_{L}\left(r_{0}, \theta_{0}\right)}{G_{L}\left(r, \theta_{0}\right)}
$$

No ponto de referência, $r_{0}=1 \mathrm{~cm} \mathrm{e} \theta_{0}=90^{\circ}$, o valor da função de dose radial é 1 . Devido ao alto gradiente de dose em regiões muito próximas à fonte de Braquiterapia, é difícil a determinação experimental desse parâmetro. Nesse sentido, o método mais recomendável é a determinação através de simulação Monte Carlo.

\section{Função de Anisotropia}

A função de anisotropia $[F(r, \theta)]$ descreve a anisotropia da distribuição de dose em um meio ao redor da fonte de radiação. Fisicamente, ela inclui os efeitos de espalhamento e atenuação, descrevendo a variação da dose em função dos ângulos relativos ao plano transverso. A função de anisotropia pode ser matematicamente calculada através da equação (7):

$$
F(r, \theta)=\frac{\dot{D}(r, \theta)}{\dot{D}\left(r, \theta_{0}\right)} \frac{G_{L}\left(r, \theta_{0}\right)}{G_{L}(r, \theta)}
$$

À medida que a espessura do encapsulamento da fonte aumenta, bem como a energia do fóton diminui ou o ângulo aproxima-se de $0^{\circ}$ e $180^{\circ}$, o valor da função de anisotropia diminui [2]. Assim como na função de dose radial, devido ao alto gradiente de dose nas proximidades da fonte de radiação, é recomendado o uso de simulação Monte Carlo para determinação desse parâmetro dosimétrico.

\section{Modelagem da fonte com o pacote PENELOPE}

A fonte de ${ }^{192} \mathrm{Ir}$ é um emissor gama e possui aproximadamente 17 linhas de emissão, onde o valor médio é de $397 \mathrm{keV}$ [23]. O espectro de radiação primário usado nas simulações, foi composto pelas 17 linhas mais prováveis de $65 \mathrm{keV}$ à $1378 \mathrm{keV}$ [24]. A geometria da fonte clínica de ${ }^{192} \mathrm{Ir}$, Varian modelo GammaMed Plus, foi modelada consistido em um cilindro de parte ativa de ${ }^{192} \mathrm{Ir}$ com $0,35 \mathrm{~cm}$ de comprimento e $0,03 \mathrm{~cm}$ de raio. $\mathrm{O}$ encapsulamento da fonte também em formato cilindro, feito de aço inoxidável AISI $316 \mathrm{~L}$, com $0,5 \mathrm{~cm}$ de comprimento e $0,055 \mathrm{~cm}$ de raio. $\mathrm{O}$ cabo da fonte, também feito de aço inoxidável AISI 316L, com $150 \mathrm{~cm}$ de comprimento e $0,055 \mathrm{~cm}$ de raio [25]. A geometria e todas as dimensões da fonte de ${ }^{192}$ Ir são mostradas na figura 2. 


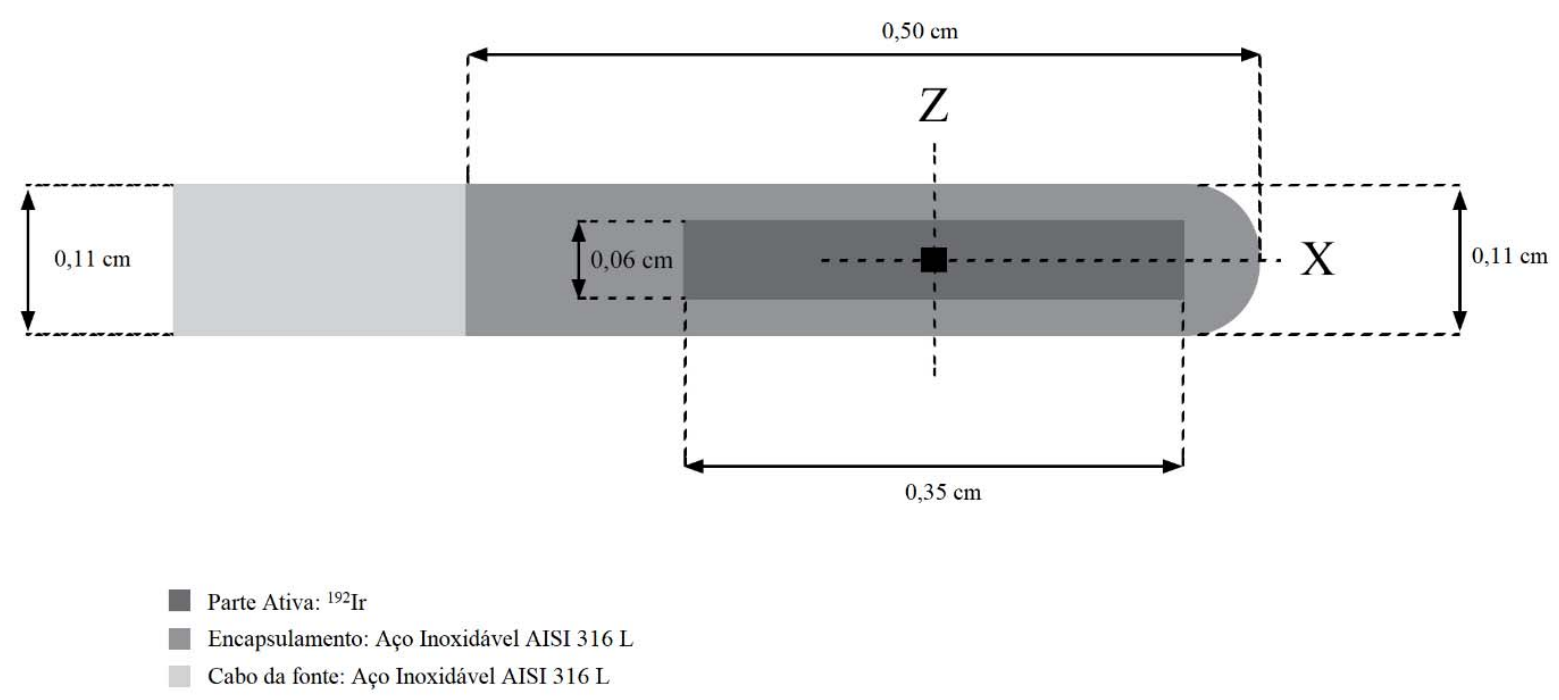

Figura 2: Geometria e dimensões da fonte de ${ }^{192}$ Ir de Braquiterapia usada nesse trabalho.

Nas simulações, os dados das seções de choque dos materiais foram obtidos junto ao banco de dados do PENELOPE, enquanto que para o aço inoxidável AISI $316 \mathrm{~L}$, um arquivo de material específico foi criado utilizando as frações de peso dos materiais através da referência [25].

Para a realização das simulações desse trabalho, foi realizado a paralelização do pacote PENELOPE de acordo com Badal e Sempau [26]. Nas simulações foi utilizado um cluster com 8 processadores, pertencentes ao CeTi-RP (Centro de Tecnologia e Informação de Ribeirão Preto) na Universidade de São Paulo, onde cada núcleo possui memória de 16 gigabytes em cada nó e processador Intel i7. Em todas as simulações, a fonte foi posicionada no centro de um objeto simulador cúbico de $30 \mathrm{~cm}$ de lado. Para registro de informações dosimétricas, foi selecionada uma distância de $10 \mathrm{~cm}$ nas três direções dos eixos cartesianos, que foram divididas em 51 elementos de volume de $0,20 \mathrm{~cm}$ de aresta. O número de partículas primárias simuladas permaneceu constante em $10^{9}$ em cada um dos oitos processadores; a energia de corte para fótons e elétrons foi de $100 \mathrm{keV}$ e os parâmetros de histórias condensadas $(\mathrm{C} 1$ e $\mathrm{C} 2)$ foram fixados em 0,3 , otimizando o tempo das simulações. $\mathrm{O}$ parâmetro $\mathrm{C} 1$ está relacionado à deflexão angular média de partículas carregadas $\mathrm{e}$ o parâmetro $\mathrm{C} 2$ está relacionado à perda fracional de energia entre os eventos fortes consecutivos.

\section{Sistema de Planejamento BrachyVision}

Os valores de dose relativa na direção radial da fonte, obtidos utilizando simulação Monte Carlo com o pacote PENELOPE, foram comparados com o sistema de planejamento BrachyVision, pertencente ao Hospital das Clínicas da Faculdade de Medicina de Ribeirão Preto da Universidade de São Paulo. Foi simulado no sistema de planejamento um objeto simulador homogêneo com as mesmas dimensões ao utilizado no pacote PENELOPE. A fonte de radiação foi posicionada no centro do mesmo e foram obtidos perfis de dose nos mesmos três planos da simulação. Os valores das doses foram normalizados no primeiro elemento de volume fora da fonte de Braquiterapia e foram comparados com os dados computacionais utilizando o pacote PENELOPE.

\section{RESULTADOS E DISCUSSÃO}

\section{Fator Geométrico}

O fator geométrico foi determinado analiticamente de acordo com as equações 3 à 5 . Na figura 3 são apresentados os fatores geométricos (a) de $0,3 \mathrm{~cm}$ à $1,0 \mathrm{~cm}$ e (b) de $2,0 \mathrm{~cm}$ à $5,0 \mathrm{~cm}$. 


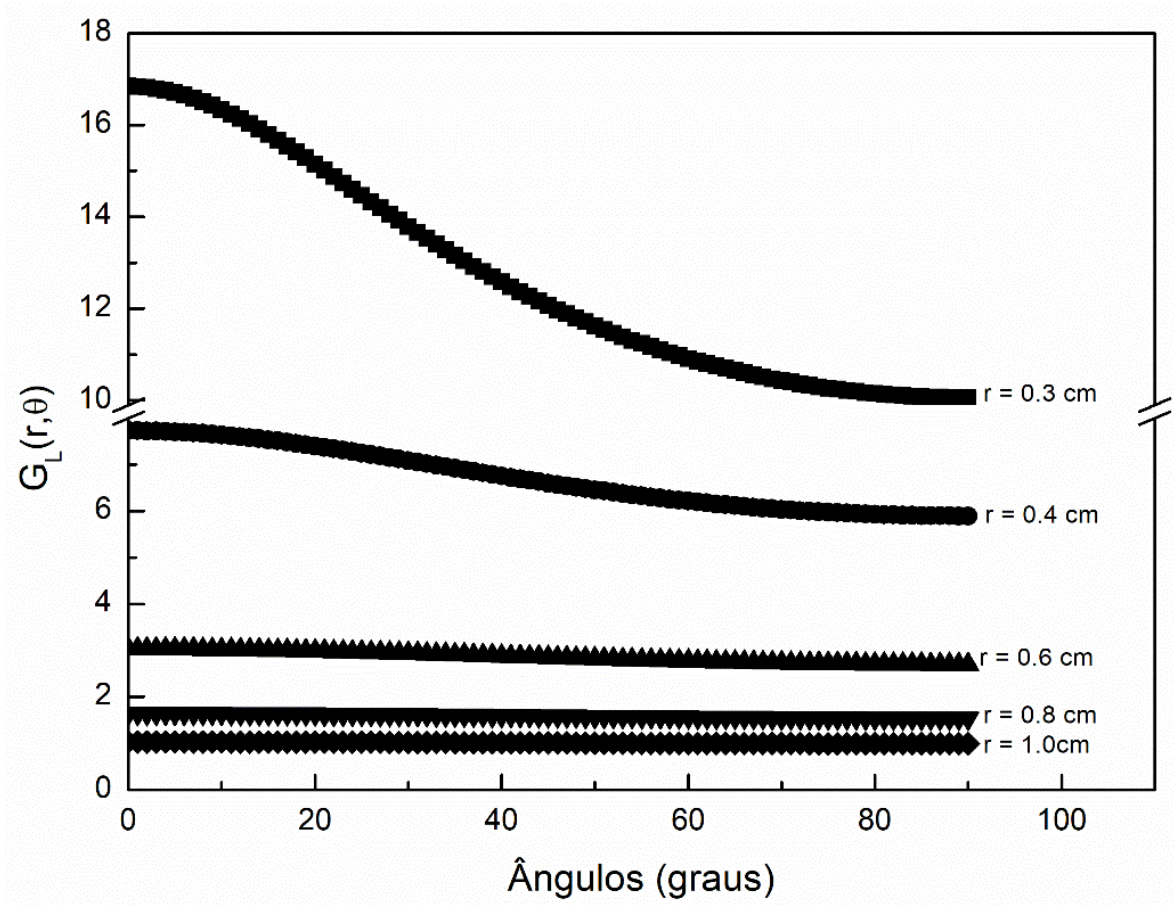

(a)

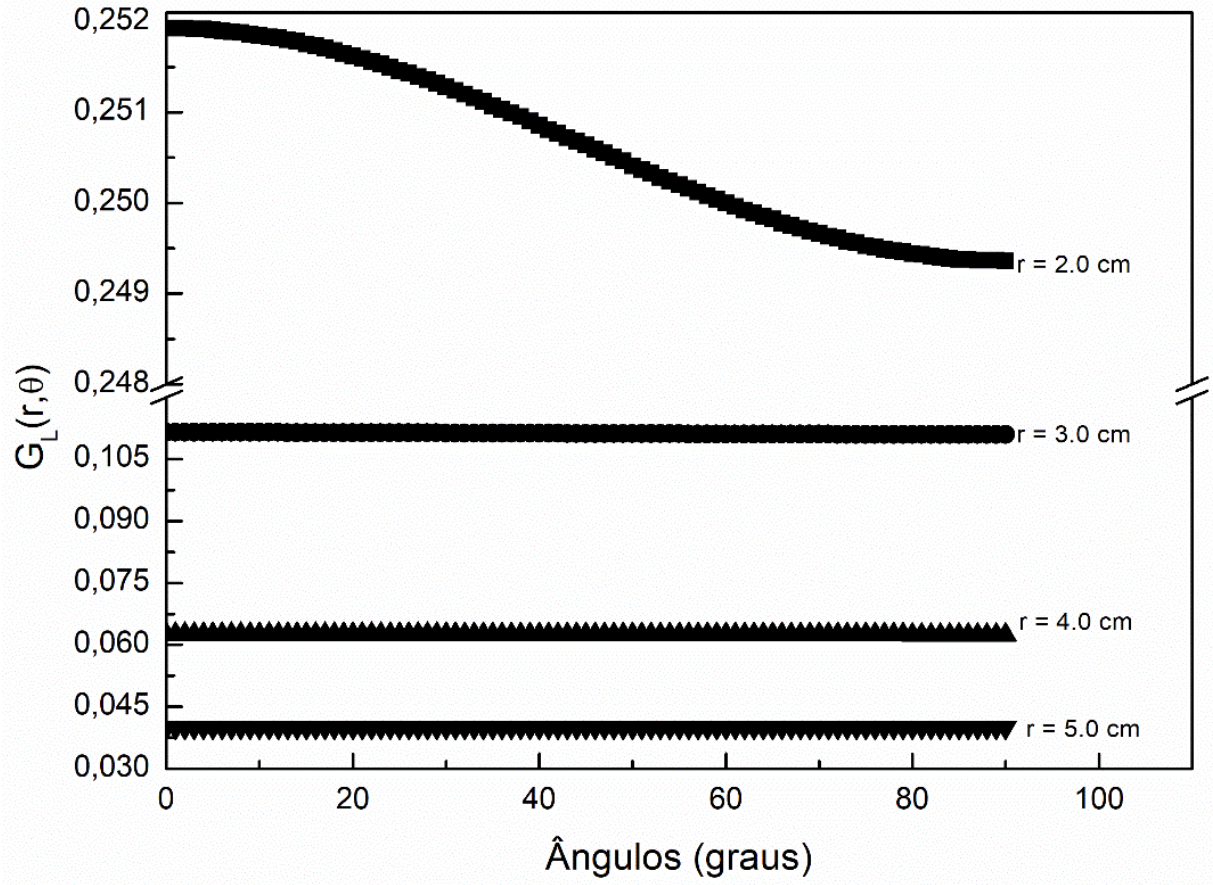

(b)

Figura 3: Fator geométrico para cada ângulo (a) de 0,3 à 1,0 cm e (b) de 2,0 $\mathrm{cm}$ à $5,0 \mathrm{~cm}$.

Como é possível observar através da figura 3 (a) e (b), o fator geométrico é máximo no topo do encapsulamento e no cabo da fonte, que são representados pelos ângulos $0^{\circ} \mathrm{e} 180^{\circ}$, respectivamente. No intervalo de $0^{\circ}$ à $90^{\circ}$, o fator geométrico é decrescente, atingindo seu menor valor no plano transverso $\left(90^{\circ}\right)$. De $90^{\circ}$ à $180^{\circ}$, o fator geométrico cresce de forma simétrica ao intervalo de $0^{\circ}$ à $90^{\circ}$, atingindo novamente o maior valor em $180^{\circ}$. O fator geométrico leva em consideração a distribuição do material radioativo da fonte, não contabilizando o efeito do encapsulamento.

Para distâncias maiores com relação ao centro da fonte, esse valor tende a zero, uma vez que essa correção é baseada na Lei do Inverso dos Quadrados das Distâncias, considerando uma aproximação linear da fonte. 


\section{Função de Dose Radial}

Esse parâmetro foi obtido usando simulação Monte Carlo com o pacote PENELOPE, para distâncias de $0,3 \mathrm{~cm}$ à $5,0 \mathrm{~cm}$ do centro da fonte. Na figura 4 é apresentada a função de dose radial para todas as distâncias analisadas.

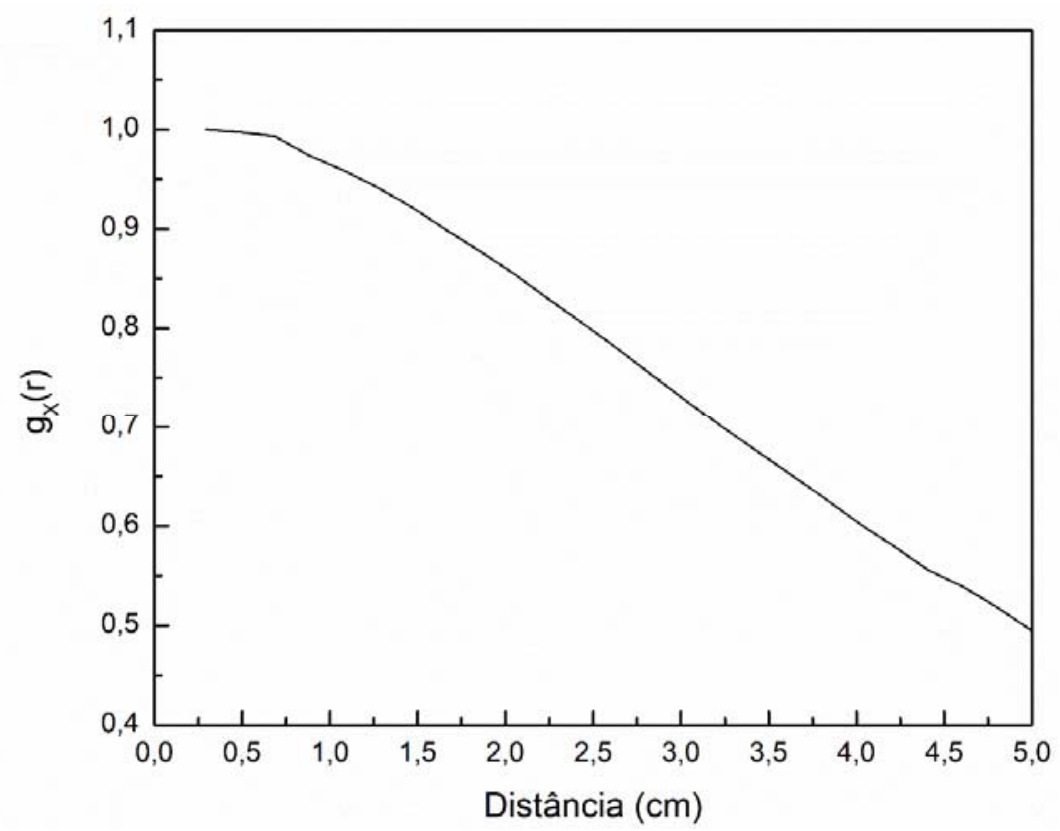

Figura 4: Função de dose radial obtida computacionalmente para a fonte de ${ }^{192}$ Ir para distâncias de 0,3 $\mathrm{cm}$ à $5 \mathrm{~cm}$ do centro da fonte.

A determinação desse parâmetro dosimétrico pode ser realizada experimentalmente, porém é recomendado o uso de simulação Monte Carlo devido ao alto gradiente de dose em regiões próximas à fonte de radiação e a dificuldade de posicionamento de dosímetros nessas regiões. $\mathrm{Na}$ tabela 1 são mostrados os valores da função de dose radial para distâncias de $0,3 \mathrm{~cm}$ à $5 \mathrm{~cm}$ do centro da fonte.

Tabela 1: Função de dose radial para distâncias de $0,3 \mathrm{~cm}$ à $5 \mathrm{~cm}$ do centro da fonte.

\begin{tabular}{cc}
\hline Distâncias (cm) & $\begin{array}{c}\text { Função de Dose } \\
\text { Radial (gx) }\end{array}$ \\
\hline 0,3 & 1,0000 \\
0,5 & 0,9975 \\
0,7 & 0,9933 \\
0,9 & 0,9739 \\
1 & 0,9590 \\
2 & 0,8537 \\
3 & 0,7253 \\
4 & 0,6021 \\
5 & 0,4950 \\
\hline
\end{tabular}

Estudos têm mostrado que o tamanho do objeto simulador utilizado para a determinação computacional da função de dose radial, pode influenciar nos resultados. Karaiskos e colaboradores [27] mostraram resultados para essa função calculada computacionalmente, utilizando objetos simuladores com diâmetros de $10 \mathrm{~cm}$ à $50 \mathrm{~cm}$. Quando os dados são comparados entre si, foram encontradas diferenças de até $25 \%$ próximo à borda dos objetos simuladores. De acordo com os 
autores, o espalhamento precisa ser levado em consideração e em objetos simuladores menores há uma redução na contribuição desse fator próximo às bordas.

\section{Função de Anisotropia}

Os valores da função de anisotropia foram determinados computacionalmente através da equação (7). Na figura 5 são mostrados os valores da função de anisotropia, obtidos nesse trabalho, utilizando o pacote PENELOPE.

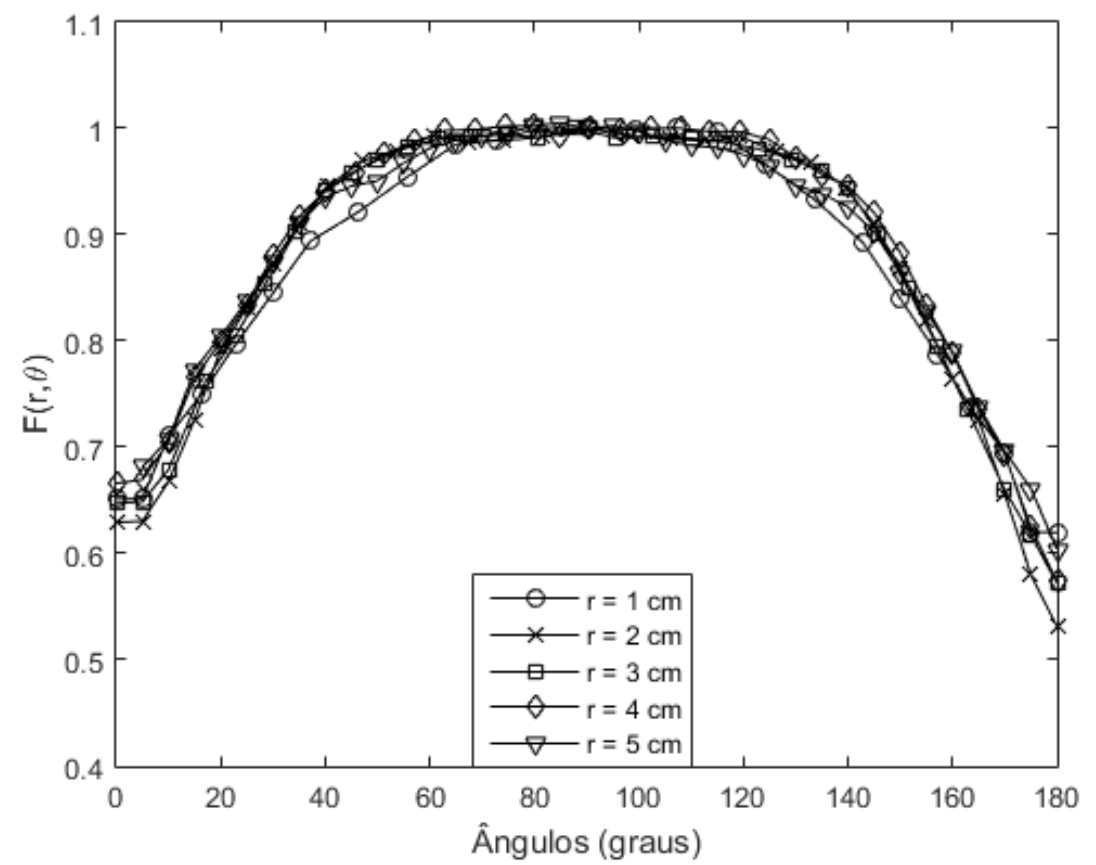

Figura 5: Função de anisotropia para distâncias de $1 \mathrm{~cm}$ à $5 \mathrm{~cm}$, obtidas computacionalmente, para a fonte de ${ }^{192} I r$.

A função de anisotropia não é simétrica entre $0^{\circ}$ e $180^{\circ}$ devido à ausência de simetria da fonte com relação ao eixo transverso, do centro ao topo do encapsulamento e do centro ao cabo da fonte de radiação. Quando essa função se aproxima do topo do encapsulamento, o ângulo aproxima-se de $0^{\circ}$ e à medida que essa função se aproxima do cabo, o ângulo tende à $180^{\circ}$. Os valores da função de anisotropia para ângulos selecionados, para as distâncias de $1 \mathrm{~cm}$ à $5 \mathrm{~cm}$, são mostrados na tabela 2.

Para distância mais próximas à fonte $(\mathrm{r}=1 \mathrm{e} \mathrm{r}=2 \mathrm{~cm})$ como mostrado na figura 5, as oscilações entre os valores dessa função são maiores devido ao tamanho do elemento de volume, utilizado para armazenar as informações de dose, combinado aos parâmetros computacionais utilizados na simulação, bem como energias de absorção de fótons e elétrons e parâmetros de condensação de histórias, como mostrado por Anwar e colaboradores [6]. Outros autores também encontraram flutuações em regiões próximas à fonte, como apresentado por Ballester e colaboradores, que calcularam a função de anisotropia para distâncias a partir de $0,75 \mathrm{~cm}$ do centro da fonte, utilizando BrachyDose, Geant4, MCNP5, MCNP6 e PENELOPE [28]. Comparando os valores da função de anisotropia entre esses pacotes, Ballester e colaboradores encontraram diferenças de até $30 \%$, principalmente quando os ângulos de aproximam do topo do encapsulamento e do cabo $\left(0^{\circ}\right.$ e 180 , respectivamente).

Assim como foi mencionado para a função de dose radial, diferentes tamanhos de objetos simuladores podem apresentar diferentes valores de função de anisotropia ou até mesmo grandes incertezas, devido à contribuição do espalhamento. A maior incerteza obtida nas simulações da função de anisotropia nesse trabalho foi de $4 \%$, para a distância de $1 \mathrm{~cm}$. 
Tabela 2: Função de Anisotropia para alguns ângulos para as distâncias de $1 \mathrm{~cm}$ à $5 \mathrm{~cm}$.

\begin{tabular}{cccccc}
\hline $\begin{array}{c}\text { Ângulos } \\
\text { (graus) / } \\
\text { Distâncias } \\
\text { (cm) }\end{array}$ & $\mathrm{r}=1 \mathrm{~cm}$ & $\mathrm{r}=2 \mathrm{~cm}$ & $\mathrm{r}=3 \mathrm{~cm}$ & $\mathrm{r}=4 \mathrm{~cm}$ & $\mathrm{r}=5 \mathrm{~cm}$ \\
\hline $\mathbf{0}$ & & & & & \\
$\mathbf{1 0}$ & 0,6513 & 0,6294 & 0,6474 & 0,6662 & 0,6817 \\
$\mathbf{2 0}$ & 0,7106 & 0,6678 & 0,6791 & 0,7052 & 0,7074 \\
$\mathbf{3 0}$ & 0,7969 & 0,7918 & 0,7623 & 0,7998 & 0,8047 \\
$\mathbf{4 0}$ & 0,8453 & 0,8721 & 0,8539 & 0,8808 & 0,8736 \\
$\mathbf{5 0}$ & 0,8941 & 0,9461 & 0,9422 & 0,9410 & 0,9353 \\
$\mathbf{6 0}$ & 0,9219 & 0,9698 & 0,9702 & 0,9764 & 0,9509 \\
$\mathbf{7 0}$ & 0,9546 & 0,9933 & 0,9899 & 0,9980 & 0,9815 \\
$\mathbf{8 0}$ & 0,9852 & 0,9874 & 0,9935 & 0,9987 & 0,9920 \\
$\mathbf{9 0}$ & 0,9991 & 0,9919 & 0,9918 & 1,0020 & 1,0030 \\
$\mathbf{1 0 0}$ & 1,0000 & 1,0000 & 1,0000 & 1,0000 & 1,0000 \\
$\mathbf{1 1 0}$ & 0,9991 & 0,9929 & 0,9920 & 1,0002 & 0,9974 \\
$\mathbf{1 2 0}$ & 1,0001 & 0,9882 & 0,9909 & 0,9971 & 0,9838 \\
$\mathbf{1 3 0}$ & 0,9660 & 0,9926 & 0,9807 & 0,9972 & 0,9754 \\
$\mathbf{1 4 0}$ & 0,9332 & 0,9684 & 0,9714 & 0,9720 & 0,9466 \\
$\mathbf{1 5 0}$ & 0,8927 & 0,9447 & 0,9435 & 0,9458 & 0,9261 \\
$\mathbf{1 6 0}$ & 0,8399 & 0,8680 & 0,8491 & 0,8832 & 0,8640 \\
$\mathbf{1 7 0}$ & 0,7369 & 0,7649 & 0,7356 & 0,7882 & 0,7901 \\
$\mathbf{1 8 0}$ & 0,6947 & 0,6564 & 0,6598 & 0,6932 & 0,6962 \\
& 0,6199 & 0,5313 & 0,5721 & 0,5736 & 0,6040 \\
\hline
\end{tabular}

\section{Comparações com o Sistema de Planejamento}

Os dados obtidos nesse trabalho foram comparados com dados sistema de planejamento BrachyVision, tipicamente utilizado em tratamentos de Braquiterapia, nos três diferentes planos de dose avaliados $(z=0, z=+0,4 \mathrm{~cm} \mathrm{e} z=-0,4 \mathrm{~cm})$. Os valores de dose foram normalizados no primeiro ponto fora da fonte em todos os planos estudados, tanto para as simulações quanto para $\mathrm{o}$ sistema de planejamento. Os perfis de dose simulados e calculados pelo sistema de planejamento foram obtidos nos mesmos planos, facilitando sua comparação. Da mesma forma, os valores de dose da simulação também foram normalizados no primeiro ponto fora da fonte. Na figura 6 são mostradas as comparações das doses relativas percentuais ao ponto mais próximo da fonte entre o sistema de planejamento BrachyVision e a simulação com o pacote PENELOPE para os planos (a) $\mathrm{z}=-0,4 \mathrm{~cm},(\mathrm{~b}) \mathrm{z}=0 \mathrm{~cm}$ e (c) $\mathrm{z}=0,4 \mathrm{~cm}$, respectivamente. Em (d) são apresentadas as diferenças absolutas, em pontos percentuais, entre os dados do sistema de planejamento e obtidos com simulação Monte Carlo.

A maior incerteza obtida para as simulações com o pacote PENELOPE foi de $0,3 \%$ no plano central da fonte $(\mathrm{z}=0 \mathrm{~cm})$ para distância mais próxima da fonte. Comparando-se as doses relativas obtidas com o pacote PENELOPE e o sistema de planejamento, a maior diferença encontrada foi de aproximadamente 2 pontos percentuais para o plano mais próximo do cabo $(\mathrm{z}=-0,4 \mathrm{~cm})$ à $8,9 \mathrm{~mm}$ do centro da fonte. 

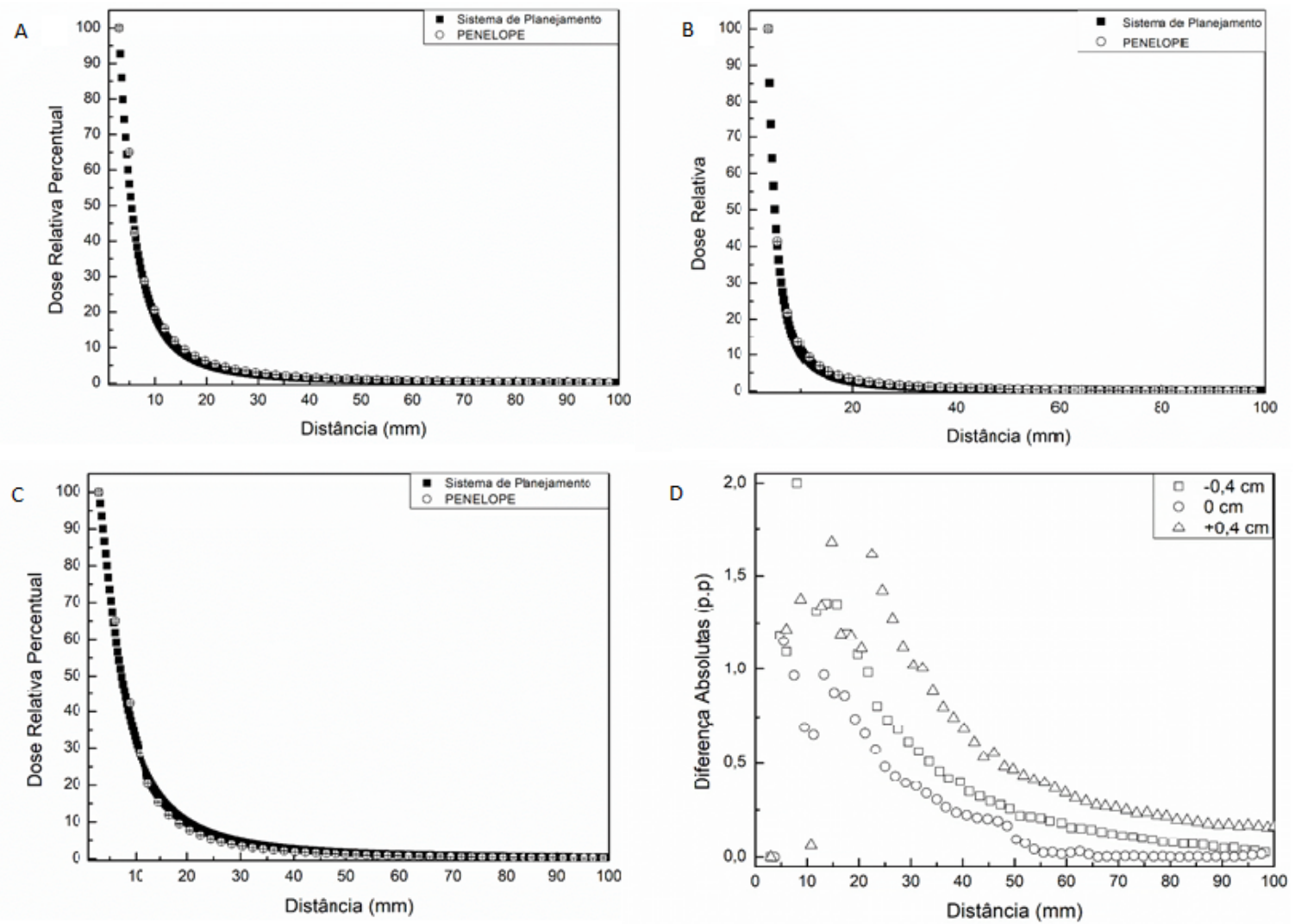

Figura 6: Doses relativas percentuais ao ponto mais próximo da fonte, para o sistema de planejamento BrachyVision e simulação Monte Carlo com o pacote PENELOPE para os planos (a) $z=-0,4 \mathrm{~cm}$,

(b) $z=0 \mathrm{~cm} \mathrm{e} \mathrm{(c)} z=+0,4 \mathrm{~cm}$. Em (d) são apresentadas as diferenças absolutas, em pontos percentuais, entre os dados do sistema de planejamento e obtidos com simulação Monte Carlo.

Através das comparações realizadas em três diferentes planos é possível verificar em os dados obtidos nas simulações com o pacote PENELOPE estão em concordância satisfatória (menor que $2 \%$ ) com os dados obtidos junto ao sistema de planejamento, indicando que o pacote PENELOPE tem grande potencial na determinação de parâmetros dosimétricos de fontes de braquiterapia de alta taxa de dose.

\section{CONCLUSÃO}

Devido ao alto gradiente de dose em regiões próximas à fonte de braquiterapia é difícil a determinação experimental de parâmetros dosimétricos para esse tipo de fonte, sendo indicado o uso de simulação Monte Carlo para a determinação desses parâmetros. Nesse trabalho, foram determinados os parâmetros dosimétricos de uma fonte clínica de braquiterapia de alta taxa de dose em regiões próximas à fonte de irradiação. A função geométrica foi determinada analiticamente, enquanto que a função de dose radial e a função de anisotropia foram determinadas computacionalmente, utilizando simulação Monte Carlo com o pacote PENELOPE. Devido à combinação de parâmetros de entrada utilizado nas simulações, os dados obtidos nesse trabalho apresentaram menores flutuações quando comparados a outros estudos reportados na literatura.

As doses relativas obtidas com o PENELOPE e com o sistema de planejamento apresentaram concordância de $98 \%$ no intervalo de distâncias estudadas. Assim, o pacote PENELOPE apresentase como ferramenta promissora em cálculos de parâmetros dosimétricos em fontes de braquiterapia de alta taxa de dose. 


\section{AGRADECIMENTOS}

Os autores agradecem ao Hospital das Clínicas da Faculdade de Medicina de Ribeirão Preto da Universidade de São Paulo pelo suporte técnico e a CAPES pelo suporte financeiro.

\section{REFERÊNCIAS BIBLIOGRÁFICAS}

1. Nath R, Anderson LL, Luxton G, Weaver KA, Williamson JF, Meigooni AS. Dosimetry of interstitial brachytherapy sources: recommendations of the AAPM radiation therapy committee Task Group No. 43. Med Phys. 1995;22(2):209-34, doi:10.1118/1.597458.

2. Rivard MJ, Coursey BM, DeWerdm LA, Hanson WF, Hug MS, Ibbott GS, et al. Update of AAPM Task Group No. 43 Report: A revised AAPM protocol for Brachytherapy dose calculations. Med Phys. 2004;31(3):633-674, doi:10.1118/1.1646040.

3. Sadeghi M, Saidi O, Tenreiro, C. Dosimetric Characteristics of Brachytherapy Sources based on Monte Carlo Method. In: Mode C. J. Applications of Monte Carlo Methods in Biology, Medicine and Other Fields of Science. India: InTech; 2011.

4. Strohmaior D, Zwierzchowski G. Comparison of 60 Co and 192Ir sources in HDR Brachytherapy. J Contemp Brachytherapy. 2011;3(4):199-208, doi:https://doi.org/10.5114/jcb.2011.26471.

5. Safigholi H, Meigooni AS, Song WY. Comparison of 192Ir, 169Yr, and 60Co High-Dose-Rate Brachytherapy Sources for Skin Cancer Treatment. Med Phys. 2017;44(9):4426-4436, doi:10.1002/mp.12335.

6. Anwarul IM. Akramuzzaman MM, Zakaria GA. EGSnrc Monte Carlo-aided dosimetric studies of the new BEBIG 60Co HDR brachyrherapy source. J Contemp Brachytherapy. 2013;5(3):148-156, doi:https://doi.org/10.5114/jcb.2013.37419.

7. Reed JL, Rivard MJ, Micka JA, Culberson WS, DeWerd LA. Experimental and Monte Carlo dosimetric characteriation of a $1 \mathrm{~cm}$ (103)Pd brachytherapy source. Brachytherapy. 2014;13(6):657-667, doi:http://dx.doi.org/10.1016/j.brachy.2014.04.001.

8. Lemarechal Y, Bert J, Falconnet C, Després P, Valeri A, Schick U, et al. GGEMS-Brachy: GPU GEant4based Monte Carlo simulation for brachytherapy applications. Phys Med Biol. 2015;60(13):4987-5006, doi:10.1088/0031-9155/60/13/4987.

9. Pakravan D, Ghorbani M, Meigooni AS. Evaluation of 101Rh as a brachytherapy source. J Contemp Brachytherapy. 2015;7(2):171-180, doi:https://doi.org/10.5114/jcb.2015.50662.

10. Baghani HR, Lohrabian V, Aghamiri MR, Robatjazi M. Monte Carlo determination of dosimetric parameters of a new (125)I Brachytherapy source according to AAPM TG-43 (U1) Protocol. Arch Iran Med. 2016;19(3):186-191.

11. Almansa JF, Guerrero R, Torres J, Lallena AM. Monte Carlo Dosimetric Characterization of the Flexisource Co-60 High-Dose-Rate Brachytherapy Source Using PENELOPE. Bracytherapy. 2017;16:1073-1080, doi:10.1016/j.brachy.2017.04.245.

12. Champion C, Incerti S, Perrot Y, Delorme R, Bordage MC, Bardiès M, et al. Dose Point Kernels in Liquid Water: An Intra-Comparison Between GEANT4-DNA and a Variety of Monte Carlo Codes. Appl Radiat Isot. 2014;83:137-141, doi:10.1016/j.apradiso.2013.01.037.

13. Forster RA, Cox LJ, Barrett RF, Booth TE, Briesmeister JF, Brown FB, et al. MCNP version 5. Nucl Instrum Meth B. 2004;213:82-86, doi:https://doi.org/10.1016/S0168-583X(03)01538-6.

14. Sempau J, Fernández-Varea JM, Acosta E, Salvat F. Experimental Bechmarks of the Monte Carlo code PENELOPE. Nucl Instrum Meth B. 2003;207:107-23, doi:http://doi.org/10.1016/S0168-583X(03)004531.

15. Sarrut D, Bardies M, Boussion N, Freud N, Jan S, Létang JM, et al. A review of the use and potential of the GATE Monte Carlo simulation code for radiation therapy and dosimetry applications. Med Phys. 2014;41(6):064301, doi:10.1118/1.4871617.

16. Candela-Juan C, Perez-Calatatud J, Ballester F, Rivard MJ. Calculated organ doses using Monte Carlo simulations in a reference male phantom undergoing HDR Brachytherapy applied to localized prostate carcinoma. Med Phys. 2013;40(3):033901-1-033901-10, doi:10.1118/1.4791647.

17. Wooten CE, Randall M, Edwards J, Aryal P, Luo W, Feddock J. Implementation and Early Clinical Results Utilizing Cs-131 Permanent Interstitial Implants or Gynecologic Malignancies. Gynecol Oncol. 2014;133(2):268-273, doi:10.1016/j.ygyno.2014.02.015.

18. Li Y, Tian Z, Song T, Wu Z, Liu Y, Jiang S, Jia X. A new approach to integrate GPU-based Monte Carlo Simulation into Inverse Treatment Plan Optimization for Proton Therapy. Phys Med Biol. 2017;62:289305, doi:10.1088/1361-6560/62/1/289. 
19. Beld E, Seevinck PR, Lagendijk JJW, Viergever MA, Moerland MA. Monte Carlo Study of the Impact of a Magnetic Field on the Dose Distribution in a MRI-guided HDR Brachytherapy Using Ir-192. Phys Med Biol. 2016;61:6791-6807, doi:10.1088/0031-9155/61/18/6791.

20. Chi Y, Tian Z, Jia X. Modeling Parameterized Geometry in GPU-based Monte Carlo Particle Transport Simulatin for Radiotherapy. Phys Med Biol. 2016;61:5851-5867, doi:10.1088/0031-9155/61/15/5851.

21. Rivard MJ, Ballester F, Butler WM, DeWerd LA, Ibbott, GS, Meigooni AS, Melhus CS, Mitch MG, Nath R, Papagiannis P. Supplement 2 for the 2004 Update of the AAPM Task Group No. 43: Report: Joint Recommendations by the AAPM and GEC-ESTRO. Med Phys. 2017;44(9):e297-e338, doi:10.1002/mp.12430.

22. Rivard MJ. Refinements to the geometry factor used in the AAPM Task Group Report No. 43 necessary for brachytherapy dosimetry calculations. American Association of Physicists in Medicine. Med Phys. 1999;26(11):2445-2450, doi:10.1118/1.598764.

23. Goestsch SJ, Attix FH, Pearson DW, Thomadsen BR. Calibration of 192Ir high-dose-rate after loading systems. Med Phys. 1991;18(3):462-7, doi:10.1118/1.596649.

24. Zilio VL, Joneja OP, Popowski Y, Chawla R. Dosimetric characterization of radioactive sources employed in prostate cancer therapy. 2004;3(4):201-14, doi:https://doi.org/10.1016/j.brachy.2004.09.006.

25. Baltas D, Giannouli S, Garbi A, Diakonos F, Geramani K, Ioannidis GT, et al. Application of the Monte Carlo integration (MCI) method for calculation of the anisotropy of 192Ir brachytherapy sources. Phys Med Biol. 1998;43(6):1783-1801.

26. Badal A, Sempau J. A package of Linux scripts for the parallelization of Monte Carlo simulations. Computer Physics Communications 2006;176:440-450, doi:https://doi.org/10.1016/j.cpc.2006.05.009.

27. Karaiskos P. Angelopoulos A, Sakelliou L, Sandilos P, Antypas C, Vlachos L, Koutsouveli E. Monte Carlo and TLD dosimetry of and 192Ir high dose-rate brachytherapy source. Med Phys. 1998;25(10):1975-1984, doi:10.1118/1.598371.

28. Ballester F, Tedgren AC, Granero D, Haworth A, Mourtada F, Fonseca GP et al. A generic high-dose rate 192Ir brachytherapy source for evaluation of model-based dose calculations beyond the TG-43 formalism. Med Phys. 2015;42(6):3048-3061, doi:10.1118/1.4921020. 